\title{
ERA-PLANET THE EUROPEAN NETWORK FOR OBSERVING OUR CHANGING PLANET
}

\author{
N. Pirrone ${ }^{\text {a, }}$, S. Cinnirella ${ }^{\text {b }}$, S. Nativi ${ }^{\text {c }}$, F. Sprovieri ${ }^{\text {b }}$, I.M. Hedgecock ${ }^{\text {b }}$ \\ ${ }^{a}$ CNR-Institute of Atmospheric Pollution, Rome, Italy - pirrone@iia.cnr.it \\ ${ }^{\mathrm{b}}$ CNR-Institute of Atmospheric Pollution, Rende, Italy \\ ${ }^{\mathrm{c}}$ CNR-Institute of Atmospheric Pollution, Firenze, Italy
}

\section{Commission VI, WG VI/4}

KEY WORDS: Earth observation, in-situ, policy, interoperability

\begin{abstract}
:
In the last decade a significant number of projects and programmes in different domains of Earth Observation and environmental monitoring have generated a substantial amount of data and knowledge on different aspects related to environmental quality and sustainability. Big data generated by in-situ or satellite platforms are being collected and archived with a plethora of systems and instruments making difficult the sharing of data and transfer of knowledge to stakeholders and policy makers to support key economic and societal sectors. The overarching goal of ERAPLANET is to strengthen the European Research Area in the domain of Earth Observation in coherence with the European participation in the Group on Earth Observation (GEO) and Copernicus. The expected impact is to strengthen European leadership within the forthcoming GEO 2015-2025 Work Plan. ERA-PLANET is designed to reinforce the interface with user communities, whose needs the Global Earth Observation System of Systems (GEOSS) intends to address. It will provide more accurate, comprehensive and authoritative information to policy and decision-makers in key societal benefit areas, such as Smart Cities and Resilient Societies; Resource efficiency and Environmental management; Global changes and Environmental treaties; Polar areas and Natural resources. ERA-PLANET will provide advanced decision-support tools and technologies aimed to better monitor our global environment and share the information and knowledge available in the different domains of Earth Observation.
\end{abstract}

\section{INTRODUCTION}

Sound and adaptive management of the Earth's natural resources and environmental quality requires reliable, relevant and readily accessible scientific data (Hoffmann et al., 2014). Europe maintains substantial capacities to generate and process Earth Observation (EO) and other environmental information. During the last decade the development of more powerful tools for data gathering and data sharing has increased the potential to foster the implementation of interoperable cyber infrastructures in environmental and EO sciences (Cinnirella et al., 2014; Nativi et al., 2014). This considerable amount of data has been rapidly made available to the scientific community, which has developed more analysis, methodologies, scientific assessments and has promoted technological progress. Despite these developments, however, this information is not always available in a suitable form for decision/policy makers and as a consequence society does not gain the full benefit of this by now almost ubiquitous environmental monitoring. The vast amount of collected data have been archived in various systems, often not catalogued or indexed and not available in an integrated way to the general public or to policy makers and stakeholders. More efficient synergies between datasets would enhance new and innovative scientific and technical studies as well as fostering the use of these datasets by the general public and decision makers for technology and policy development.

Recently, online data discovery and access (i.e. open data, data sharing) have become essential to enable multi- and crossdisciplinary research for both the public and decision makers.
Making the best use of existing data and ensuring information system interoperability is a new important challenge. In Europe, there is a clear need to establish a more effective interoperability between National and European research infrastructures (specifically, Copernicus and ESFRI initiatives) in order to play a more strategic role in the Group on Earth Observations (GEO) and its Global Earth Observation System of Systems (GEOSS) initiative (ESFRI, 2010; EU, 2010; Reillon, 2016). To this end a European Research Area (ERA) project was prepared with the contribution of most EO European agencies: The European network for observing our changing planet (ERA-PLANET). The European Union (EU) through ERA-PLANET may enhance its role in GEOSS by fostering a better cross- and interconnection of individual EO systems ensuring more efficient access and exploitation of datasets. Pursuing interoperability in the exchange of data, metadata, products and services in a full, open and unrestricted manner would generate business opportunities for the private sector within the EU (SMEs, industries) leading to significant benefits to European society as a whole. To facilitate effective interoperability providing low entry barriers for the GEOSS stakeholders, it is important to recognize the peculiarities characterizing the large variety of Community of Practices and their specific requirements. GEOSS recognized the need of mediation and brokering services, successfully implemented by the GEOSS Common Infrastructure (GCI) (Nativi et al. 2015). Recognising these challenges and the need for GEO-related coordination mechanisms at European and national levels, as well as possible synergies between the GEOSS and the EU

\footnotetext{
* Corresponding author
} 
Copernicus programme, ERA-PLANET is designed to reinforce the European leadership within the GEO 2015-2025 Work Plan. ERA-PLANET therefore, aims to bring together and strengthen the European, national and regional R\&I programmes in the domain of EO. The overarching goal of ERA-PLANET is the development of a Transnational Environmental Observation System in Support of European \& International Policies through the Integration of real-time monitoring data from various platforms, modelling tools and advanced global cyberinfrastructure for data sharing and interoperability. This short communication is aimed to illustrate how ERA-PLANET will contribute to EO advance in Europe.

\section{SPECIFIC OBJECTIVES}

Specific objectives of ERA-PLANET are:

1. To prepare and launch a two-stage joint transnational call structured along the following Strands:

a. Strand 1 - Smart cities and resilient societies addressing issues such as urban growth, air quality, disasters, health, contaminated sites.

b. Strand 2 - Resource efficiency and environmental management including water, energy, food security, biodiversity.

c. Strand 3 - Global change and Environmental treaties addressing global observing systems for toxic and persistent pollutants, harmonization of monitoring and coupled atmosphere-ocean-terrestrial models, evaluation of ecosystem response to regional/global emission changes, support to policy implementation. Climate forcing is one of the major variables to be considered in assessing global change patterns and analysis of policy scenarios.

d. Strand 4 - Polar areas and natural resources in a highly climate-sensitive regions including the evaluation of the impact of energy resource exploitation, the impact of long-range transport of air pollutants and their atmospheric deposition, airsurface exchange mechanisms, and environmental pressure from increasing anthropogenic activity in areas with sensitive ecosystems.

2. To fund projects according to a priority list set by external experts.

3. To monitor funded projects and to report progress accordingly.

4. To develop a strategic research agenda to reinforce the ERA and to coordinate the cross- and inter-cooperation of European and national programs in key and selected EO domains.

5. To coordinate initiatives and promote best practices and guidelines with the aim to improve the interoperability among the existing and future projects on EO and links to the GCI.

The project objectives exhibit the capacity of ERA-PLANET to reply to the key challenge of the call and specifically aim to simplify access to the information required by decision makers and bring together and strengthen the European national and regional research and innovation programmes in the EO domain. ERA-PLANET will attempt to go beyond the coordination of EO during its implementation and will establish initiatives to survive after the duration of the project.

The project will benefit from the outcome of on-going activities being carried out by several partners in the framework of international, national and regional programmes (Table in section B3.4) including but not limited to:
- UNEP Global Partnership on Atmospheric Mercury Transport and Fate Research (UNEP F\&T) (www.chem.unep.ch);

- Global Monitoring Plan (GMP) of the Stockholm Convention on Persistent Organic Pollutants (POPs) of UNEP (www.pops-gmp.org/);

- Task Force on Hemispheric Transport of Air Pollutants (TF HTAP) of the UNECE-LRTAP convention (www.htap.org);

- GEO Tasks and Flagships (e.g., GMOS, GEO-BON, GEOGLAM, Geohazard Supersites, GD-02 "GCI Operation"; GD-07 "GCI Development"; GD-09 "GEOSS Knowledge Base" HE-02 "Tracking Pollutants", ID-02 "Developing Institutional and Individual Capacity", EN-01 "Energy and Geo resources assessment", SB-05 "Impact Assessment of Human activities", WA-01-C3 "Cold Regions", SB-04-C1 "Global Urban Observation and Information", just to cite a few);

- ESFRI/I3 Research Infrastructures (e.g. ACTRIS- Aerosols, Clouds, and Trace gases Research InfraStructure Network, LTER, LifeWatch, EPOS, ANAEE, SIOS);

- National polar research programmes (e.g., Italian, Swedish, German) involved in measurements devoted to climate forcing, biodiversity, ecosystem management, biogeochemical cycles of heavy metals in polar ecosystems and evaluation of response times and mechanisms to changes in anthropogenic pressures and stressors.

The results of past and on-going EU funded projects (i.e., EGIDA, GMOS, EuroGEOSS, GEOWOW, MEDINA, GeoViQua, OBSERVE, BalkanGEOnet, EnviroGRIDS, EU BON, IASON, EOPOWER, BEYOND, EUCAARI, PEGASOS, AFROMAISON, BACCHUS, GEOCARBON, PEEX, CRYOLAND) and other projects related to GEOSS and Copernicus developed within the framework of a number of GEO Tasks will provide a solid basis of knowledge in terms of state-of-the-art atmospheric models, monitoring methodologies, QA/QC systems, interoperable management tools and environmental policy analysis instruments and exploitation of Copernicus services. ERA-PLANET will get benefit from the outcomes of on-going projects such as ENVRIPLUS because the overarching goal of these projects is to bring together networks on Environmental and Earth System Research Infrastructures and build common synergic solutions.

The overall scope of ERA-PLANET is to foster and support the development of comprehensive and sustainable global environmental observation and information systems, which will contribute to GEOSS activity in the EO domain. To achieve this the overarching goal of ERA-PLANET will be to prepare, launch and process a joint transnational call for proposals to select the best innovative and competitive projects that may better contribute to GEO in relation to key aspects of urban area management, exploitation and use of natural resources, implementation and verification of EU directives and international treaties related to persistent chemical pollutants in the environment, bearing in mind how they impact on human health and sensitive ecosystems including the Earth's atmosphere, oceans, cryosphere and landscapes. This global system can stimulate smart use of resources, support evidencebased policies, and provide new market opportunities through free and open access to data. ERA-PLANET will underpin research and innovation activities to answer the need of a Global Earth Observation Infrastructure, not necessarily concentrated into a single system, but inevitably federated (is federated used in this context??) through a brokering system. Research and innovation activities will be driven through a bottom up approach and addressed to the development of brokered data infrastructures, the involvement of SMEs on 
specific technological tasks in each project will allow ERAPLANET to shorten the time to market of new innovative technological solutions.

The selected projects for the four ERA-PLANET Strands will have to reinforce European participation within GEO and should also provide a research and innovation component to better bridge GEOSS with Copernicus.

To ensure a tangible contribution to GEOSS and Copernicus, ERA-PLANET is structured to have the GEOSS Work Programmes and relevant Copernicus services as its foundation. The strategic priorities laid out in the GEO Strategic Plan 20162025 and Copernicus Regulation defining the actions towards Copernicus uptake. (Is this sentence missing a verb??)

The GEOSS Strategic Plan 2016-2025 is structured on the basis of three strategic objectives: (a) advocacy of EO as the foundation of environmental information; (b) engagement with stakeholders to address every-day societal challenges, and (c) delivery of critical data, information and knowledge to inform decision-making. Along these lines the contribution of ERAPLANET to GEOSS will be realized through the:

- endorsement of full and open access to EO data;

- promotion of the use of key data management principles, as well as common standards and interoperability arrangements;

- encouragement and actions to increase the contribution of regional resources directly to the GEOSS Data-CORE and Copernicus data portal;

- engagement with key stakeholders to identify the needs in observations, and environmental and socio-economic data analyses, which can yield advances in many Societal Benefit Areas (SBAs);

- broadening of the GEOSS and Copernicus user base through well-targeted dissemination and exploitation actions;

- ensuring access to data, information and knowledge, while increasingly promoting interoperability among multiple sources of data; and deliver the tools, knowledge and services suitable for intelligent exploitation by user communities;

- showcasing concrete collaborative schemes (through the strands) relying on the integration of regional capacities and skills towards addressing specific challenge priorities.

The Copernicus programme provides users with free, full and open access to environmental data, including direct access to satellite data. Established services have reached different degrees of maturity. Some are already operational (land monitoring and emergency management) while others are still in a pre-operational mode (atmospheric monitoring and marine monitoring) or in a development phase (climate change monitoring and services for security applications).

Copernicus is considered as a European contribution to building GEOSS (see the the European Parliament and of the Council establishing the Copernicus Programme and repealing Regulation (EU) No 911/2010). ERA-PLANET can foster data and service sharing technologies based on the brokering approach. Since Copernicus is user-driven, it requires the continuous, effective involvement of users, particularly regarding the definition and validation of service requirements. This requirement is reflected in ERA-PLANET's specific target to improve societal participation in EO initiatives.

Apart from ERA-PLANET's structural alignment with GEOSS and Copernicus described above, an Advisory Board comprised of GEO and Copernicus, European Commission Representatives, other European interest organizations (EEA, ESA, ECMWF); Representatives of JPIs, GEOSS and Copernicus Secretariat; Representatives of international programmes and organizations. A bi-lateral cooperative agreement with ESA is under discussion between ESA and the ERA-PLANET consortium. This Board will enable ERAPLANET to be proactive in ensuring that the implementation of the overall ERANET as well as the individual projects produce a tangible and substantial contribution to GEOSS and Copernicus.

As the past framework programme has strongly supported the objectives of the GEOSS 10-Year Implementation Plan and the GEO Work Plan through ad-hoc research projects, ERAPLANET is designed to ensure more cross-cutting integration in Europe for the next 5 years in order to further advance the implementation and use of Global Environmental Datasets and the information that can be derived from them. One of the main goals of ERA-PLANET is to ensure a closer cooperation between Copernicus and GEOSS, therefore addressing specifically the achievement of the expected impact of the Call which is an "effective coordination mechanism and integration of major European research and innovation Earth Observation programme". The ERA-PLANET partners are frequently engaged in both Copernicus and GEOSS, and often at both the programmatic and project delivery level, so that they are uniquely well placed to see that this desired coordination and integration happens.

\section{IMPLEMENTATION}

ERA-PLANET will prepare and launch a joint transnational call structured along the four major strands (Figure 1). Each proposal will be required to recognize and introduce a set of Architectural Principles for Data and Services Interoperability set as Guidelines in ERA-PLANET (see also Figure 2 for Horizontal activities in each project funded under the Strands). Each Strand will, therefore, be represented by a single project. A two-step call will be adopted in ERA-PLANET to ensure a real competition and prioritise ideas. This procedure is necessary to ensure that only entities that are eligible for funding under the national programmes involved in the joint are invited to step 2.

Each Strand is based on a list of issues and goals that the Consortium identified. Nevertheless this list will be revised in the light of ideas received from Stage One of the evaluation, to allow for several good ideas to be further promoted.

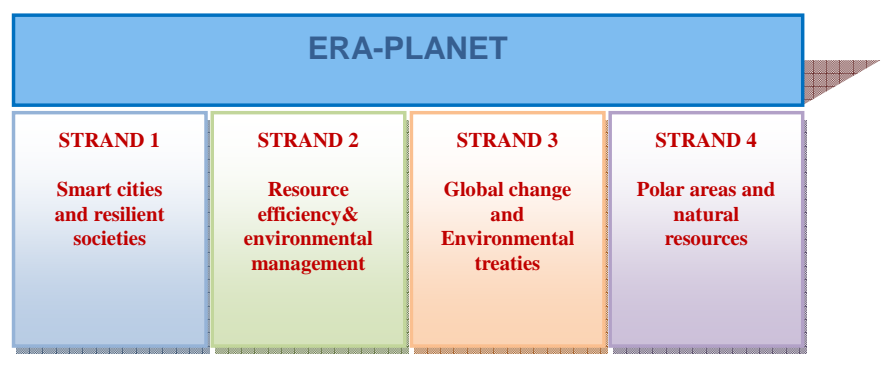

Figure 1. Strands selected for the implementation of ERAPLANET

\subsection{Strand Description}

Overall goal and objectives of Strand 1 - According to UN's "World Urbanization Prospects: The 2014 Revision" in today's increasingly global and interconnected world, over half of the world's population lives in urban areas, while the coming decades will bring further profound changes to the size and 
spatial distribution of the global population. Continuing urbanization is projected to add 2.5 billion people to the urban population by 2050 , and the proportion of the world's population living in urban areas is expected to increase to $66 \%$. Under these circumstances, sustainable development challenges will be increasingly concentrated in cities" (United Nations, Department of Economic and Social Affairs, Population Division (2014). World Urbanization Prospects: The 2014 Revision, Highlights (ST/ESA/SER.A/352)).

The overall goal of ERA-PLANET's Strand 1 is to fully exploit data and information, tools and services that derive from the increasing multi-sensor, multi-temporal and multi-scale capacity and use of EO (in situ and space-borne), in conjunction with other cross cutting observational platforms, towards identifying and addressing cities' vulnerabilities, supporting policies to design strategies and procedures to shield citizens and mitigate impacts of urbanization.

The achievement of this overarching goal will be based on two discrete, but at the same time interconnected, objectives: create Smart Cities and develop Resilient Societies. In particular:

- Bring together technology, government and society to adopt and take first steps towards the Smart Cities model, to create sustainable economic development and high quality of life, by optimizing the use of $\mathrm{EO}$ in conjunction with innovative observational/modelling platforms and digital technologies and by applying excellent practices in key areas such as the environment, protection of human health, health care, energy, governance and mobility.

- Increase the resilience of societies living in cities by building dedicated capacities to prepare for, withstand and respond faster to stresses and new challenges of growing urban environments.

Of course cities are complex environments in both economic, governance and societal terms and there might be numerous factors that may influence their ability to continue to function properly and in a coordinated fashion, in the face of daunting and/or unexpected challenges, as a result of urbanisation, climate change or other potential stressors. Moreover, different cities may need to weigh vulnerabilities differently, based on the specific particularities they bear, and finally focus on different aspects that would help them bounce back rapidly from disruptions, exploiting advantages and assets already existing and/or applied. In this context, Strand-1 will put emphasis on specific issues namely urban growth, air quality and urban microclimate, natural and manmade disasters, health, contaminated sites, and suggest specific case studies that present increasing interest in terms of physical processes (e.g. multiple and discrete pollution sources contributing to elevated pollution levels, complex terrains), urban morphologies and structural organisation, high and uncontrolled urbanization growth rates and vulnerability to climate change or the on-going economic crisis, the latter also resulting to behavioural changes. This list of issues will be revised on the basis of input received during the Stage-One of the Call for proposals.

Overall goal and objectives of Strand 2 - The Earth provides all of the resources that humans and other species require to live on the planet; it is the only source that we have at the present time. With world population at 7 Billion and continuing to grow, these resources are under significant pressure. This pressure is exacerbated by the rapid economic development of countries that did not formerly consume significant resources, which also happen to be some of the most populous (Brazil, Russia, India, China, South Africa). In and of itself, this development is of course a good thing, but it has the potential to result in continued pressure on resources for decades to come, even if the global population were to stabilise rapidly in the coming years. This continuous pressure affects water, energy, biodiversity and food resources as well as security. Water, food and energy are most often linked in the so-called nexus, but biodiversity is directly affected by the pressures on water, food and energy. If water, energy and food security are to be achieved and simultaneously their impact on biodiversity minimised, decision-makers, including those responsible for only a single sector, need to consider broader influences and cross-sectorial impacts. A nexus approach to sectorial management, through enhanced dialogue, collaboration and coordination, is needed to ensure that co-benefits and trade-offs are considered and that appropriate safeguards are put in place. A nexus perspective increases the understanding of the interdependencies across the water, energy and food sectors and influences policies in other areas of concern such as climate and biodiversity.

The overall goal of ERA-PLANET's Strand-2 is to fully exploit data and information, tools and services that derive from the increasing multi-sensor, multi-temporal and multi-scale capacity and use of EO (in situ and space-borne), in conjunction with other cross cutting observational platforms, towards a nexus approach, to evaluate, predict and monitor the resources, and promote cross-sectorial approaches to reconcile resource efficiency and environmental management ensuring human development according to the sustainable development goals.

The achievement of this overarching goal will be based on two discrete, but at the same time interconnected, objectives: resource efficiency and environmental management in a nexus perspective. In particular:

- Improve resources assessment and efficiency in a sectorial approach, to create the necessary knowledge to evaluate, predict and monitor the resources and to build crosssectorial approaches, by optimizing the use of EO in conjunction with innovative observational/modelling platforms and digital technologies.

- Develop and promote cross-sectorial approaches to reconcile resource efficiency and environmental management to propose approaches, methods and tools for stakeholders and decision-makers for developing mutually beneficial responses and enhancing the potential for cooperation between and among all sectors.

The resources under most pressure include water, soil, energy, minerals, availability of sunlight and darkness, biodiversity of natural species, cultivated crops, animals, fish and other species, as well as the land and oceans they live on and the air they breathe. For all of these resources there are two linked challenges facing society: (a) security of supply, to sustain economic growth, promote wealth, end poverty and hunger; and (b) management of their extraction, cultivation and utilisation in a sustainable fashion, to preserve the environment for future generations. Strand-2 considers both of these challenges, because they are also high on the policy agenda, being addressed by many relevant programmes including GEO.

The policy and programme review carried out in WP-6 will identify the most critical topics from the European perspective amongst all of these global challenges in security of supply and environmental management. These will then be the subject of Strand-2 project to be selected through the ERA-PLANET Call.

Overall goal and objectives of Strand 3 - During the last two decades progress has been made in the understanding of the cycling of persistent pollutants between different environmental compartments. Climate variability is considered one of the major drivers influencing the transport patterns of highly toxic and persistent pollutants from the emission sources to aquatic 
and terrestrial receptors. Several conventions and international programs such as the World Meteorological Organization's Global Atmosphere Watch (GAW), the European Monitoring and Evaluation Programme (EMEP) supporting the CLRTAP, the GMOS supporting the Minamata Convention, the Global Monitoring Plan (GMP) supporting the Stockholm Convention and various Research Infrastructure (ESFRI) initiatives of the European Commission have made substantial efforts to establish data centres and quality control programs to enhance integration of atmospheric composition measurements from different national and regional networks, and to establish observational sites in under-sampled, remote regions around the world.

In order to support the implementation of international environmental policy there is a need for globally distributed observational data for major persistent pollutants. The availability of observational data that are representative of emission source regions, or which intercept major transport patterns with changing meteorological and climate conditions would improve capacity for the validation of regional and global scale models used to analyse different socio-economic scenarios of emission control and environmental quality control. In the past 5 years in the framework of the GEO - Task HE-02 "Tracking Pollutants" global observation systems for mercury and POPs were developed to support the achievement of the goals of GEOSS and other on-going international Programs (i.e., UNEP Mercury Program) and Conventions (i.e., Minamata, Stockholm Convention, UNECE-LRTAP TF HTAP). Specifically a coordinated global mercury $(\mathrm{Hg})$ network has been established within GMOS (www.gmos.eu) as has the Global Monitoring Programme (GMP) for POPs. In order to fulfil the need for data sharing and interoperability, in the framework of the GEO-Task IN-03 "Common Infrastructures" a user-friendly GCI was designed and developed to support and sustain the operational activities of these global networks allowing users to search and access GEOSS resources (e.g. datasets and services).

The overall goal of Strand-3 is to develop a new paradigm for real-time monitoring of the quality of our environment with reference to the contamination of air, water and terrestrial ecosystems by persistent pollutants. The overarching objective of this strand is the development of a fully integrated system of advanced sensors (based on nano-structured advanced materials) for major persistent pollutants coupled with state-ofthe-art interoperable systems for data sharing and data management. Currently the prohibitive investment and management/maintenance costs of monitoring systems for these pollutants limit our capacity to develop sustainable observational systems for long-term monitoring programmes. The aim of Strand-3 is to produce new advanced monitoring technologies to increase the geographical coverage (number of monitoring sites) of our current global monitoring networks for persistent pollutants at rural (and background) sites as well as in contaminated (highly impacted) areas that coupled with advanced GCI will support policy makers and stakeholders in the implementation of major international conventions and programmes.

ERA-PLANET responds to a demand from the GEO and from the Global Atmosphere Watch (GAW) Program of WMO to improve the global observation system for the main atmospheric pollutants, including short-lived and long-lived climate forcers such as aerosol and trace gases and persistent chemical pollutants such as mercury $(\mathrm{Hg})$ or Persistent Organic Pollutants (POPs). In Europe, networks for atmospheric observation have been developed to respond to international conventions (i.e., Minamata, UNECE-LRTAP TF HTAP) or to a demand from the research communities (EU Research Infrastructures). While there is a clear need for a global approach, the integration of regional initiatives into a global framework remains problematic and will require additional support in the future (GAW report \#207).

Overall goal and objectives of Strand 4 - Polar Regions, and more generally regions of the cryosphere, play a key role in the Earth's climate system and are changing currently at an unexpected and unprecedented rate. Regional sea level change, the release of greenhouse gases as a result of warming permafrost soils, the alteration of fresh water resources are major societal challenges in Europe and worldwide. High latitude areas harbour with particularly fragile ecosystems, where pollutants may have severe impacts on ecosystem services. Pollutant emissions are typically not very high in these regions however pollutant cycling is markedly different such as the potential for remobilisation of anthropogenic pollution from secondary cryospheric sources. Even though pollutants are mainly transported from mid-latitudes, Arctic pollutants are now already being produced locally in the Arctic (shipping, oil/gas extraction, increasing urbanization and tourism).

Pollutant loads to ecosystems and the cryosphere are likely to increase due to climate change and economic drivers. Monitoring pollutants (i.e. $\mathrm{Hg}$, POPs, Black Carbon, SOx, NOx, VOCs, PM) and some emerging pollutants is of fundamental importance in understanding how the inputs have and will change, and how the processes which govern their cycling function in the cryosphere. A combination of remote sensing, in-situ EO and modelling are the key to understand the drivers and processes leading to changing ice sheets and glaciers.

The goal is to cooperate with on-going initiatives and existing networks which measure atmospheric composition and its temporal changes (i.e., short-lived climate pollutants as e.g. ozone, aerosols and their precursors) and networks more focused on targeted chemical pollutants (i.e., GMOS, NADP) that are more persistent in the environment and have a significant impact on human health (i.e., PAHs, $\mathrm{Hg}$, selected POPs, heavy metals) which are all subject to rigorous QA/QC protocols. This framework should combine a wealth of EO data from ground, airborne, ship and satellite platforms to create an environment in which the links between pollutant concentrations in different ecosystem compartments can be investigated, and the causal relationship between pollutant emissions and ecosystem impacts can be understood. It will provide data to an integrated cyber-infrastructure to understand the changes in atmospheric composition and pollutant deposition, how pollutant cycling changes over time, and how these changes impact ecosystems and human health.

Understanding how pollutant inputs and cycling change, and will continue to change in the cryosphere, requires a combination of complex monitoring systems to obtain relevant environmental information. An interoperable system which allows researchers to access all the necessary information required to ascertain the links between contaminant levels, meteorological conditions, climate forcing, and ecosystem health indicators is required. To achieve this it will be necessary to compare and contrast pollutant concentrations and distributions with, for example, data concerning atmospheric composition, and snow and ice characteristics such as albedo and temperature. To understand pollutant biogeochemical cycles with changing climate, EO and modelling of processes in snow, sea-ice, glaciers, ice-sheet or permafrost will be needed. The choice of contaminants to be monitored is extremely important for the relevance of the project, and should include 
$\mathrm{Hg}$, legacy pesticides, flame retardants, emerging POPs (in accordance with the Stockholm Convention), heavy metals and aerosols.

To use available data sets of present atmospheric pollution in the right perspective, it is necessary to set a baseline of contaminant levels in the past (historical records). For this reason it is extremely important to look at different environmental archives and to reconstruct past atmospheric composition through environmental proxies.

Combining pollutant and environmental data with information on changes in ecosystem services, particularly habitat, provisioning and regulatory should provide a holistic view of how the cryosphere will respond under different contamination and climate future scenarios.

\subsection{Key-Enabling Technologies}

The fast progress in digital information technologies and development of high resolution sensors and tools puts new challenges to the EO domain, especially in the context of "Big Data" (i.e. Data-intensive Science), and "Open Science". For disciplinary and domain applications, systems interoperability largely depends on the adoption and implementation of agreed digital data technologies, standards, and specifications with a disciplinary/domain service bus or other means of information exchange, if available. Since ERA-PLANET is addressing multi- and inter-disciplinary Communities, that require to deal with extremely attention to interoperability among the diverse (existing) e-infrastructures and information systems developed and sustained at the national and European level. This is pursued by adopting more flexible and sustainable approaches. Another important action concerns the coordination of the many national and regional activities aiming to develop einfrastructures and information systems dealing with EO data, information, knowledge, and documents.

The following Figure 2 illustrates the importance of einfrastructure and interoperability themes for ERA-PLANET. Along with Data Quality and Policy, e-infrastructures and interoperability are essential to access and share (e.g. use) the resources (including data, services, knowledge bases, documents, sensors, and models) provided by many projects and initiatives at the national and European levels. They are the Key Enabling Technologies (KETs), the funded ERA-PLANET projects will utilize to work out their cross- and interdisciplinary research and reinforce the European role in GEOSS

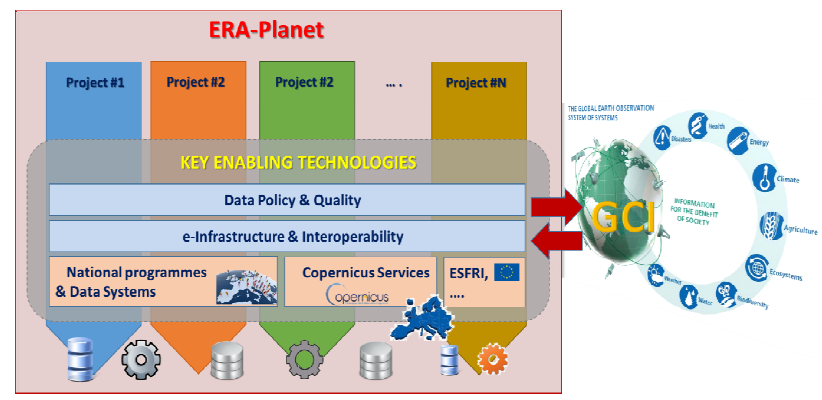

Figure 2. Common Activities in ERA-PLANET Strands by using Key Enabling Technologies

In order to assure that all funded projects within ERA-PLANET will adopt state-of-the-art KETs, ERA-PLANET will establish specific Working Groups on KETs (i.e., on Data Policy and
Quality, e-Infrastructure \& Interoperability) which will set up the guidelines to be adopted by all four selected projects within ERA-PLANET.

The overarching horizontal layers common to all projects under ERA-PLANET aim to coordinate and advance the sector of the KETs for EO applications to reinforce the European role in GEOSS and guide dialogue with Copernicus downstream services. ERA-PLANET though its WGs on KETs will recognize and introduce a set of Architectural Principles for Data and Services Interoperability to be applied by all the proposal. Besides, ERA-PLANET will issue a set of Guidelines to implement such Principles (e.g. the DAB guidelines); the selected proposals will be asked to follow these guidelines as set by expert WGs on KETs.

As to the activities of the horizontal layers, ERA-PLANET will consider tasks to pursue a set of goals that are more general and some objectives that are more specific. The main general (long term) goals include:

- Improve interoperability among the existing and next coming digital infrastructures at the National and European level.

- Reinforce the European role in GEOSS as to interoperability with the GCI and other relevant Global programmes.

- Re-use and advance the European capacities, improving the European competitiveness in the IT and Data Science sectors.

- Support Open Science, GEOSS Data Sharing principles, and GEOSS Data Management principles.

- Support the need to share more information and less data e.g., the GEOSS Essential Variables.

- Develop integrated sets of semantic and terminological resources covering all the knowledge sub-domains (i.e., thesauri, taxonomies, lexicons and ontologies).

- The more specific (short-medium term) objectives include:

- Improve interoperability amongst observational, modelling, data assimilation and prediction systems to maximise value and benefits of EO investments.

- Assure and coordinate the interoperability of the enabling technologies developed/utilized/advanced by the specific projects that will be funded by the ERA-PLANET.

- Provide a useful guidance on and good practice for the enabling technology, at the European and National levels, to the specific projects that will be funded by the ERAPLANET.

- To promote a synergic and complementary advancement of the enabling technologies for the access and re-use (i.e. the interoperability) of EO resources (e.g., data, knowledge, services, models).

- Enable cross and inter-disciplinary research at the ERAPLANET level, through EO resources interoperability.

\section{ENGAGEMENT OF PROGRAMME OWNERS \& MANAGERS}

In its initial stages ERA-PLANET's strategy is to initiate and develop relationships with Programme Owners \& Programme Managers, which are stakeholders of the project. To go beyond the simple information/outreach process ERA-PLANET plans to empower them to be active in the decision making through the adoption of a multiple-stage process by:

- Informing stakeholders about ERA-PLANET objectives and how it can strengthen the European Research Area in the domain of Earth Observation at both EU and national levels; 
- Involving Stakeholders to ensure that issues and concerns about such objectives are understood and considered as part of the decision-making process;

- Collaborating with Stakeholders in formulating options and in providing recommendation;

Adopting recommendations in formulating the two-stage Transnational Call for proposals

\section{CONSORTIUM AS A WHOLE}

A large effort was put on building the Consortium to involve the most active European Public Bodies in the field of EO (Figure 3). They range from Institutions involved in Earth monitoring through satellites (e.g., FMI, FZJ, CNR, GFZ, NOA, SRI, TROPOS) to those that manage large monitoring networks (e.g., CNR, AWI, CNRS, FMI, MfN, MU, NOA, UHEL). To implement and achieve the project's objectives (i.e., implementation of each project based on Strands) the Consortium embraces the widest scientific community melted to address themes of four Strands. Most partners have a long history of collaboration that makes more feasible the ERAPLANET management. A number of them have also a proactive participation in building GEOSS. ????Also the infrastructural and interoperability aspects have been considered relevant as the coordination the enabling technologies for EO applications is a key ERA-PLANET success????. Some are leading Institutions in GEOSS that are strongly supporting the GCI implementation, while others make available their competencies in semantic interoperability and information management. Similarly, several partners have been active in the institutions and development aspects of building GEOSS, and include a co-chair of the relevant implementation board.

Finally, in order to fulfil the objectives of ERA-PLANET it is important to have a consortium where the financial resources (in term of in-kind and cash contribution) offered to the project from the participant institutions are considerable (> 66\% of topup EU funding). In addition to the highly trained professionals they include resources invested in on-going monitoring efforts (logistics and laboratory costs), Information Technology developments and current capacity building programmes.

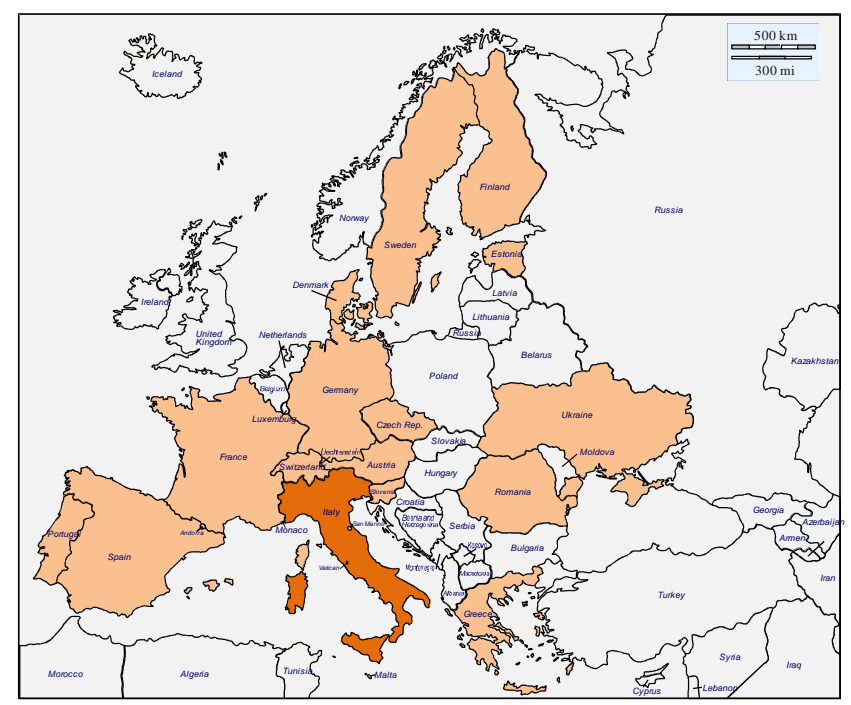

Figure 3. Countries participating to ERA-PLANET

\section{REFERENCES}

Cinnirella, S., D'Amore, F., Bencardino, M., Sprovieri, F. and Pirrone, N., 2014. The GMOS cyber(e)-infrastructure: advanced services for supporting science and policy. Environmental Science and Pollution Research, 21(6), pp. 4193-4208.

ESFRI, 2010. ESFRI Regional Issues Working Group. Report 2010. Available at: https://ec.europa.eu/research/infrastructures /pdf/regional_issues.pdf

$\mathrm{EU}$, 2010. Communication from the Commission to the European Parliament, the Council, the European Economic and Social Committee and the Committee of the Regions Europe 2020 Flagship Initiative Innovation Union SEC(2010) 1161. Available at: http://eur-lex.europa.eu/legal-content/EN /ALL/?uri=CELEX\%3A52010DC0546

Hoffmann, A., 2014. Improved access to integrated biodiversity data for science, practice, and policy - the European Biodiversity Observation Network (EU BON). Nature Conservation, 6: pp. 49-65.

Nativi, S., Mazzetti, P., Craglia, M., Pirrone, N., 2014. The GEOSS solution for enabling data interoperability and integrative research. Environmental Science and Pollution Research, 21(6), pp. 4177-4192.

Nativi, S., Mazzetti, P., Santoro, M., Papeschi, F., Craglia, M., Ochiai, O., 2015. Big Data challenges in building the Global Earth Observation System of Systems. Environmental Modelling \& Software, 68(June 2015), pp. 1-26.

Reillon, V., 2016. The European Research Area. Evolving concept, implementation challenges. EPRS, European Parliamentary Research Service. Available at: http://www.europarl.europa.eu/RegData/etudes/IDAN/2016/579 097/EPRS_IDA(2016)579097_EN.pdf 